\title{
Asset price dynamics in a financial market with heterogeneous trading strategies and time delays
}

\author{
Alessandro Sansone $e^{\mathrm{a}, \mathrm{b}, *}$, Giuseppe Garofalo ${ }^{\mathrm{c}, \mathrm{d}}$ \\ a Department of Economic Sciences, University of Rome "La Sapienza", Italy \\ ${ }^{\mathrm{b}}$ School of Finance and Economics, University of Technology, Sydney, NSW, Australia \\ ${ }^{\mathrm{c}}$ Department of Managerial, Technological and Quantitative Studies, University of Tuscia, Viterbo, Italy \\ ${ }^{\mathrm{d}}$ Department of Public Economics, University of Rome "La Sapienza", Italy
}

Available online 1 March 2007

\begin{abstract}
In this paper we present a continuous time dynamical model of heterogeneous agents interacting in a financial market where transactions are cleared by a market maker. The market is composed of fundamentalist, trend following and contrarian agents who process market information with different time delays. Each class of investors is characterized by path dependent risk aversion. We also allow for the possibility of evolutionary switching between trend following and contrarian strategies. We find that the system shows periodic, quasi-periodic and chaotic dynamics as well as synchronization between technical traders. Furthermore, the model is able to generate time series of returns that exhibit statistical properties similar to those of the S\&P 500 index, which is characterized by excess kurtosis, volatility clustering and long memory.
\end{abstract}

(C) 2007 Elsevier B.V. All rights reserved.

Keywords: Dynamic asset pricing; Heterogeneous agents; Complex dynamics; Chaos; Stock market dynamics

\section{Introduction}

In recent years there has been a growing disaffection with the standard paradigm of efficient markets and rational expectations. In an efficient market, asset prices are the outcome of the trading of rational agents, who forecast the expected price by exploiting all the available information and know that other traders are rational. This implies that prices must equal the fundamental values and therefore changes in prices are only caused by changes in the fundamental. In real markets, however, traders have different information on traded assets and process information differently, therefore the assumption of homogeneous rational traders may not be appropriate. The efficient market hypothesis motivates the use of random walk increments in financial time series modeling: if news about fundamentals are normally distributed, the returns on an asset will be normal as well. However, the random walk assumption does not allow the replication of some stylized facts of real financial markets, such as volatility clustering, excess kurtosis, autocorrelation in square and absolute returns,

\footnotetext{
*Corresponding author. Department of Economic Sciences, University of Rome "La Sapienza", Italy.

E-mail addresses: alessandro.sansone@fastwebnet.it (A. Sansone), giuseppe.garofalo@uniroma1.it (G. Garofalo).
} 